${ }^{1}$ Centro Universitario de la Costa Sur (CUCSUR)-Universidad de Guadalajara. Centro de Investigaciones en Comportamiento Alimentario y Nutrición (CICAN)- Centro Universitario del Sur (CUSUR)- Universidad de Guadalajara, Jalisco, México. ${ }^{2}$ Centro de Investigaciones en Comportamiento Alimentario y Nutrición (CICAN)-Centro Universitario del Sur

(CUSUR)- Universidad de Guadalajara, Jalisco, México.

${ }^{3}$ Centro Universitario de los Valles (CUVALLES)-Universidad de Guadalajara, Jalisco, México. ${ }^{a}$ Maestra en Ciencias (MsC) ${ }^{b} \mathrm{PhD}$.

Fuente de apoyo: No hubo. Los autores no declaran conflictos de intereses.

Recibido el 9 de mayo de 2017, aceptado el 11 de octubre de 2017

Correspondencia a: Carmen Livier García Flores Dirección postal: Centro de Investigaciones en Comportamiento Alimentario y Nutrición (CICAN).

Centro Universitario del Sur- Universidad de Guadalajara.

Av. Enrique Arreola Silva, Colonia Centro, 883, Edificio X. 49000, Cd Guzmán, Jal. México. carmen.garcia@cucsur.udg.mx

\section{Saciación vs saciedad: reguladores del consumo alimentario}

\author{
CARMEN LIVIER GARCÍA-FLORES ${ }^{1, \mathrm{a}}$, \\ ALMA GABRIELA MARTÍNEZ MORENO ${ }^{2, b}$, \\ CLAUDIA PATRICIA BELTRÁN MIRANDA ${ }^{2, b}$, \\ ANA PATRICIA ZEPEDA-SALVADOR ${ }^{2, a}$, \\ LAURA VANESA SOLANO SANTOS ${ }^{3, a}$
}

\section{Satiation and satiety in the regulation of energy intake}

The study of the factors that regulate high energy food intake is especially relevant nowadays due to the high prevalence of overweight and obesity. Food intake regulation can be divided in two basic processes, namely satiation and satiety. Satiation is the process that determines the moment in which feeding stops and regulates the amount of ingested food during a single meal. Satiety is the interval between meals and regulates the time elapsed between two meals. The longer the interval, the lower energy intake. Each of these processes are regulated by different factors, which are here reviewed.

(Rev Med Chile 2017; 145: 1172-1178)

Key words: Appetite Regulation; Feeding Behavior; Satiety Response.

\section{L} a alimentación es un fenómeno complejo. Su acción permite a los organismos mantener un suministro energético homeostático determinado por un mecanismo que modula la ingesta y el gasto energético, así como la frecuencia de consumo ${ }^{1}$. Dicho suministro es fundamental para la regulación del apetito y del peso corporal. Por ello, ha sido estudiado por diversas ciencias, entre ellas las biológicas y psicologicas ${ }^{2}$. Debido a la prevalencia de sobrepeso y obesidad a nivel mundial, se han estudiado los factores que promueven el elevado consumo de alimentos ricos en energía y que contribuyen al desequilibrio del balance energético ${ }^{3-6}$. Desde 1960 se ha estudiado de cerca los factores que influyen sobre la regulación de la ingesta alimentaria, entre ellos: a) socioculturales (estilo de vida, causas del consumo, situación socioeconómica); b) ambientales (apariencia, sabor, tamaño de porciones); c) biológicos (señales hormonales, contenido energético, cantidad ingerida) yd) psicológicos (influencias cognitivas, creencias, expectativas acerca de los alimentos, control voluntario del consumo) ${ }^{12}$. Por esta razón se han realizado estudios especializados en la medición del hambre, el apetito, el deseo por comer e inclusive con la sensación de plenitud del acto de comer, realizando asociaciones entre factores biológicos y psicológicos que ayudan a comprender la conducta alimentaria de los individuos, ${ }^{13}$. En este sentido, una alternativa para el estudio y la comprensión de los factores que inciden sobre la ingesta y la regulación alimentaria es diferenciar y describir los distintos procesos de saciación y saciedad ${ }^{14}$. Por lo anterior, el presente documento tiene como objetivo describir y analizar el efecto de los diversos factores que intervienen en la regulación del consumo alimentario, específicamente mediante el proceso de saciación, el cual debe ser diferenciado de la saciedad, pues a pesar de que son regidos y regulados por diferentes elementos, son utilizados y referidos indistintamente. 


\section{Procesos mediadores de la ingesta en un episodio alimentario}

Una característica fundamental para el estudio y análisis de la ingesta alimentaria es en primera instancia determinar el tipo de terminología utilizada para describir el acto de comer, por ejemplo: comida, "snack", comidas ocasionales, eventos de alimentación, momentos de alimentación y episodios de alimentación ${ }^{13}$. Este último, ha sido caracterizado por incluir siete dimensiones para su análisis: 1) tipo de alimento o bebida consumida; 2) duración; 3) procesos mentales; 4) actividades realizadas en torno al acto de comer; 5) condiciones físicas del alimento o bebida; 6) localización del lugar de consumo y 7) interacción social ${ }^{15}$. Algunos factores situacionales involucrados en el consumo alimentario que pueden ser analizados durante el período prandial, es decir, durante el proceso de saciación son: el tipo de alimento consumido, tamaño y cantidad de las porciones, el número de bocados, el inicio y el término de una comida, así como los modales. Otros factores de mayor complejidad que pueden ser analizados son los aspectos sensoriales, el tipo de nutrientes consumidos, los aspectos psicológicos, las condiciones temporales, físicas, económicas, sociales $y$ culturales ${ }^{13}$. Cabe destacar que en la ingesta de alimento o bebidas durante un episodio alimentario, se emiten señales sensoriales, cognitivas, metabólicas y hormonales en el organismo, que a su vez pueden determinar la cantidad ingerida y por ende, regular la ingesta alimentaria ${ }^{5,14}$. Dichas señales han sido catalogadas por Blundell, Rogers y Hill como "La Cascada de Saciedad" (Figura 1), que permite identificar los factores que influyen sobre el episodio alimentario. Es decir, puntualizan los elementos que determinan la saciación, en la cual actúan componentes sensoriales a corto plazo, como la variedad, textura, palatabilidad, tacto, olfacción, visión y audición de los alimentos. Por otro lado, los factores que actúan a largo plazo y que influyen para determinar el proceso de saciedad (período que inicia con el término del episodio ingestivo) son los factores cognitivos, post-ingestivos y post-absortivos ${ }^{16}$. Un componente de suma importancia involucrado en el término de la ingesta alimentaria es el grado de saciedad sensorial específica de un alimento, donde la función de la textura del alimento es de suma importancia para determinar el fin de la ingesta alimentaria e influye directamente sobre la cantidad de alimento ingerido ${ }^{5,17-22}$. El cual si bien es denominado como saciedad, en el estricto significado de la palabra, podrías ser referido como saciación sensorial específica, por llevarse a cabo durante el período ingestivo o prandial.

\section{¿Qué es la saciación?}

La saciación es el proceso que determina el momento cuando se suspende el acto de comer, es decir, la delimitación del final de un episodio alimentario que determina la cantidad de alimento ingerido ${ }^{23}$. Este a su vez se identifica por su duración a corto plazo o temprano, determinado durante aproximadamente $20 \mathrm{~min}$ desde el inicio de la ingesta alimentaria ${ }^{5}$. Se caracteriza por la intervención de factores sensoriales, la composi-

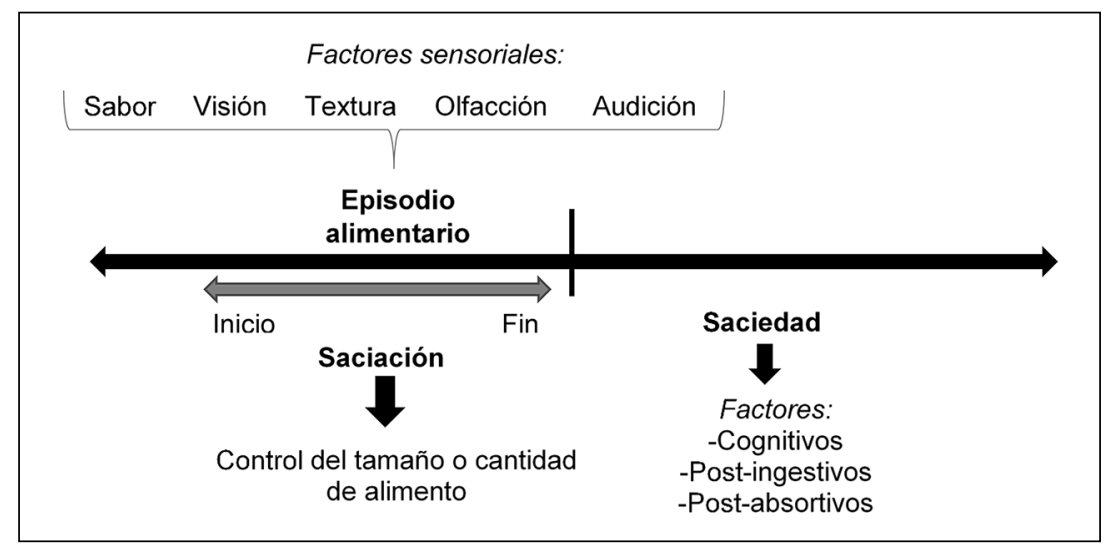

Figura 1. Procesos mediadores de la saciación durante un episodio alimentario. Muestra los factores sensoriales que intervienen y controlan el tamaño o cantidad consumida de alimento. Adaptado de "Satiation, satiety: concepts and organization of behavior", F. Bellisle and J. Blundell, 2013, Satiation, satiety and the control of food intake, theory and practice, p. 9. 
ción de macronutrientes, la densidad energética, además de los factores cognitivos y pre-absortivos tempranos ${ }^{14,24}$.

Tanto el proceso de saciedad como el de $s a$ ciación han sido utilizados indistintamente. Sin embargo, no son determinados por los mismos factores ni en el mismo lapso de tiempo durante un episodio alimentario. El proceso de saciedad se denomina como el intervalo entre comidas en función del tiempo transcurrido. Puede ser determinado por una prueba de precarga alimentaria, rangos de apetito y utilizado para predecir el nuevo evento ingestivo, además de especificar el llenado gástrico e incluso ser cuantificado por marcadores periféricos como glucosa, insulina y grelina ${ }^{5}$.

Durante el proceso de saciación interactúan diversas señales de regulación interna que disminuyen la ingesta, causado por el efecto de hormonas y neuropéptidos como colecistoquinina (CCK) sintetizada en el intestino delgado como respuesta al consumo de proteínas y grasas, cuyo efecto saciante radica en la acción de dos tipos de receptores, el primero de ellos es el CCK-1 que estimula desde las terminales aferentes vagales la transmisión nerviosa de saciedad al núcleo del tracto solitario. El segundo es el receptor del CCK2 que se localiza en el nervio vago y en el sistema nervioso central (SNC) actuando en conjunto con la colecistoquinina liberada a nivel cerebral para causar un efecto saciante. En este mismo sentido, el péptido similar al glucagon tipo 1 (GLP-1) sintetizado en el estómago, intestino y a nivel cerebral tiene un efecto saciante, disminuyendo así la ingesta alimentaria. Contrariamente la grhelina sintetizada en el estómago, intestino y cerebro tiene un efecto orexigénico, actúa principalmente en el núcleo arcuato hipotalámico y a nivel neuronal en el núcleo del tracto solitario y dorsomotor, induciendo la ingesta alimentaria. Por lo cual se espera que las concentraciones de esta hormona tras la ingesta de alimento disminuyan, contrario a los niveles elevados reportados en sujetos en período de ayuno. ${ }^{25}$

\section{Investigaciones realizadas en los procesos de saciación y saciedad}

Las investigaciones realizadas en torno a la regulación alimentaria han sido parte de un arduo trabajo desde hace varias décadas hasta la actuali- dad, destacando así las aportaciones conceptuales, experimentales y aplicadas. Específicamente en el tenor de los procesos de saciación y saciedad los primeros hallazgos se remontan hacia el año de 1950, cuando James Strang comenzó a realizar estudios para esclarecer la importancia de conocer el por qué las personas consumían lo que comían, con lo cual definió a la saciedad como el momento en el que una persona para de $\operatorname{comer}^{26}$. Hacia el año 1957, Jean Meyer definió la saciedad como "la cesación de comer”27, por su parte Guy Hollifield y William Parson comenzaron a realizar estudios de la saciedad para medir la respuesta en ratones una vez que habían sido sometidos a períodos de privación de alimento ${ }^{28}$. En 1971, con las contribuciones retomadas de Jacques Le Magnen, los investigadores David Booth y Jonh Blundell propusieron por primera vez dos mecanismos de inhibición de la ingesta alimentaria: la saciación y la saciedad ${ }^{14}$. Por su parte, David Booth en 1976 propuso de manera particular que mediante el proceso de saciación se podía determinar el tamaño de la ingesta alimentaria ${ }^{29}$. Más tarde en 1987, John Blundell, Peter Rogers y Andrew Hill propusieron el modelo de la "Cascada de Saciedad" para determinar las diferentes señales que se involucraban en la ingestión de alimentos y bebidas sobre ambos procesos ${ }^{14}$. Hoy en día se conocen los efectos de diferentes nutrientes sobre la saciación y saciedad. Holt, Brand, Petocz y Farmakalidis propusieron el Índice de Saciedad, con el cual se identificó la jerarquización de macronutrientes en el proceso de saciedad, ubicando a las proteínas como el macronutriente que confiere mayor saciedad al organismo, seguido de carbohidratos y $\operatorname{lípidos}^{30}$. Otras investigaciones realizadas en este sentido son la identificación del efecto saciante reducido de las bebidas endulzadas en comparación con los alimentos sólidos ${ }^{6,31-33}$. Asimismo, se ha intentado esclarecer los efectos del sabor, específicamente de la dulzura, sobre la saciación y saciedad, identificando así las características de los alimentos y bebidas que incentivan al aumento y disminución del consumo ${ }^{34,35}$. Otro tipo de investigaciones realizadas son las que se enfocan en el efecto de los edulcorantes artificiales sobre la regulación alimentaria evaluando el efecto de estas sustancias sobre los procesos de saciación y saciedad enfatizando sus beneficios sobre la salud, los cuales garantizan una reducción calórica para mejorar los niveles de glucosa y el control de peso; 
sin embargo, las conclusiones referentes a este efecto aún no son del todo claras ${ }^{36,37,38}$.

\section{Aspectos determinantes de la saciación y su evaluación}

Existen múltiples elementos de suma importancia en la regulación alimentaria como la densidad energética, los factores cognitivos, sensoriales, post-ingestivos y post-absortivos. Estos elementos serán reflejados en la capacidad saciante de un alimento, además de la composición de macronutrientes, contenido de fibra y el volumen de éste ${ }^{11,13}$. Dentro de los factores que juegan un importante rol en la saciación de los alimentos se encuentran los cognitivos y sensoriales. Tal es el caso de las diferencias en la percepción entre un alimento sólido y líquido. Se ha asociado la mayor capacidad saciante a los alimentos sólidos, en comparación con los líquidos, probablemente debido a las diferencias de percepción de textura oral y fácil deglusión ${ }^{5}$. Las propiedades sensoriales de los alimentos como la textura, la apariencia y el sabor han sido estudiadas por sus efectos sobre la ingesta alimentaria, ya que de ellas dependerá que un alimento sea más placentero y aceptado por el consumidor $^{39-41}$. A continuación se describen los factores sensoriales que influyen sobre la saciación y la ingesta alimentaria, además de las técnicas utilizadas para su evaluación.

\section{Textura y saciación}

Entre las características hedónicas de algunos alimentos que pueden provocar distintas respuestas ingestivas destacan los aspectos sensoriales. La comida considerada como obesigénica es caracterizada por un elevado contenido energético y suave textura. En este sentido, los atributos o características sensoriales de un alimento juegan un importante rol sobre la saciación y la ingesta alimentaria ${ }^{42}$. La textura de un alimento puede determinar la velocidad con la que se ingiere, pues un alimento suave será consumido con mayor ligereza en comparación con uno de textura dura o crujiente, evitando la detección de las respuestas de la fase cefálica en el organismo, encargadas de la respuesta fisiológica de las señales sensoriales que informan al intestino y cerebro, la presencia de nutrientes y por ende, un efecto saciante. Por lo tanto, al no existir una adecuada señalización sensorial se producirá una baja respuesta a la $s a-$ ciació $n^{43,44}$. En este sentido, existen diversos marcadores bioquímicos que pueden ser identificados en la respuesta de la fase cefálica, entre ellos el ácido gástrico, péptidos digestivos, glucosa, insulina y grelina ${ }^{43,45,46}$. Cabe destacar que el tiempo de tránsito de un alimento en la cavidad oral tiene un importante rol en la explicación del efecto de la textura sobre la ingesta alimentaria, por esta razón los alimentos con texturas suaves o líquidas, durarán menos tiempo en la cavidad oral que los alimentos sólidos o semi-sólidos, los cuales al mantenerse más tiempo en la boca producen una mayor saciación y por lo tanto, reducen la ingesta alimentaria a libre acceso ${ }^{12,47}$.

\section{Densidad energética y saciación}

La densidad energética de un alimento es caracterizada por su contenido de agua y la composición de macronutrientes. Este contenido energético es una de las características principales que determina la ingesta alimentaria y a su vez influye en los procesos de saciación y saciedad ${ }^{29}$. Los mecanismos por los cuales la densidad energética afecta la ingesta alimentaria son múltiples y complejos, ya que se involucran factores cognitivos, sensoriales, hormonales, gastrointestinales y neuronales, que tienen influencia directa sobre las características del alimento como la forma física, propiedades sensoriales, el peso o volumen del alimento, la palatabilidad y la textura, que actúan directamente en el proceso de saciación $n^{48}$.

\section{Evaluación de la saciación}

Estudios del comportamiento alimentario en humanos eventualmente utilizan valoraciones para determinar el apetito, hambre y plenitud gástrica. Sin embargo, existen una amplia gama de escalas utilizadas para valorar subjetivamente las diferentes sensaciones de apetito como el uso de las escalas análogas visuales, utilizadas para determinar la saciación de un alimento ${ }^{49}$. Uno de los procedimientos más apropiados para evaluar el proceso de saciación es el análisis microestructural de una comida. Los principales parámetros evaluados por éste, son las características propias del alimento, resaltando la cantidad de alimento consumido, energía ingerida, duración y la tasa promedio de consumo. Otro parámetro es el análisis de los bocados, la masticación, las pausas 
durante el período ingestivo y las bebidas ingeridas $^{23,50}$.

\section{Discusión}

Existen múltiples factores que se involucran en la regulación de la ingesta alimentaria, específicamente la saciación y la saciedad son procesos mediante los cuales se puede estimar el tamaño y cantidad de alimento ingerido, así como el tiempo transcurrido entre episodios ingestivos, respectivamente. En este sentido, ha resultado se especial interés el estudio de la saciedad y su medición; sin embargo, el proceso de saciación no se ha investigado con el mismo interés, aun cuando en éste se involucran factores sensoriales que pueden intervenir en la regulación alimentaria durante el período prandial. Por lo tanto, resulta de especial interés identificar el nivel de saciación de alimentos y bebidas durante un episodio ingestivo, debido a que la saciedad refleja únicamente la consecuencia del período prandial. De este modo, al esclarecer las distinciones entre ambos procesos, se logrará identificar los elementos que influyen en la ingesta alimentaria en períodos prandiales y post-prandiales ${ }^{51}$.

Autores como Wansink, Van Ittersum y Painter concuerdan con la necesidad de investigar acerca de los niveles de saciación en condiciones experimentales $^{52}$. Situación que podría contribuir a la generación de nuevas estrategias para la modificación de la conducta alimentaria, que consista en la promoción y conocimiento del efecto de los nutrientes sobre los centros de regulación de la saciación, y a su vez, contribuyan sobre el control de la cantidad de alimento ingerido durante un episodio alimentario. Otra de las futuras expectativas en la investigación sería identificar las particularidades de la población sobre la sensibilidad genética relacionada con los procesos de saciación y saciedad, así como sus implicaciones sobre la regulación del peso corporal ${ }^{53}$.

Por lo tanto, las expectativas actuales en el estudio de la regulación alimentaria y los mecanismos involucrados en la conducta ingestiva, resultan factibles de explorar por medio de trabajos multidisciplinarios, donde se exploren y diferencien los factores que pueden determinar el consumo alimentario tanto en la saciación como en la saciedad, y por ende, intentar realizar modificaciones conductuales que permitan la prevención de problemáticas actuales de salud a nivel mundial.

\section{Conclusiones}

La saciación y la saciedad son distintos procesos, mediante su distinción se pueden esclarecer los factores que los determinan. Específicamente el estudio de la saciación resulta prometedor para identificar los factores sensoriales involucrados en la ingesta de alimento durante un episodio ingestivo, es decir, durante el período prandial. Lo cual podría contribuir al desarrollo de nuevas estrategias para la prevención del incremento de la ingesta alimentaria en la población y así evitar los efectos adversos que estos tienen sobre la salud.

Agradecimientos: Se agradece al Centro de Investigaciones en Comportamiento Alimentario y Nutrición, del Centro Universitario del Sur, Universidad de Guadalajara y al Consejo Nacional de Ciencia y Tecnología, por el apoyo otorgado con la beca número 401244 .

\section{Referencias}

1. Havel PJ. Peripheral signals conveying metabolic information to the brain: short-term and long-term regulation of food intake and energy homeostasis. Exp Biol Med 2001; 226 (11): 963-77.

2. Martínez A, López-Espinoza A, Díaz F. Modelos de regulación en conducta alimentaria. Investigación en Salud 2007; 9 (3): 172-7.

3. Moreno-Martínez M, García-Ruiz A, Sánchez-González D. Efecto de los edulcorantes no nutritivos (aspartame y sucralosa) en el peso de las ratas. Estudio prospectivo, controlado, aleatorizado, doble ciego. Rev Sanid Milit Mex 2011; 65 (4): 168-75.

4. Pérez E, Serralde A, Meléndez G. Efectos benéficos y deletéreos del consumo de fructosa. Rev Endocrinol Nutr 2007; 15 (2): 67-74.

5. Stafleu A, Zijlstra N, Hogenkamp P, Mars M. Texture and diet related behavior: a focus on satiation and satiety. En Preedy V, Watson R, Martin C, Editores. Handbook of behavior, food and nutrition. New York: Editorial Springer; 2011. p. 133-42.

6. Swithers S, Laboy A, Clark K, Cooper S, Davidson T. Experience with the high-intensity sweetener saccharin 
impairs glucose homeostasis and GLP-1 release in rats. Behavioural Brain Research 2012; 233 (1): 1-14.

7. Cassady B, Considine R, Mattes R. Beverage consumption, appetite, and energy intake: what did you expect?. Am J Clin Nutr 2012; 95 (3): 587-93.

8. DiMeglio D, Mattes R. Liquid versus solid carbohydrate: effects in food intake and body weight. Int J Obes 2000; 24 (6): 794-800.

9. Flood J, Rolls B. Soup prelods in a variety of forms reduce meal energy intake. Appetite 2007; 49 (3): 626-34.

10. Flood-Obbagy J, Rolls B. The effect of fruit in different forms on energy intake and satiety at a meal. Appetite 2009; 52 (2): 416-22.

11. Livingstone $\mathrm{M}$, Robson $\mathrm{P}$, Welch R, Burns A, Burrows $\mathrm{M}$, McCormack C. Methodological issues in the assessment of satiety. Scand J Nutr/Näringsforskning 2000; 44:98-103.

12. Zijlstra N, Mars M, Stafleu A, De Graaf C. The effect of texture differences on satiation in 3 pairs of solid foods. Appetite 2010; 55 (3): 490-7.

13. Bisogni C, Winter L, Madore E, Blake C, Jastran M, Sobal J, et al Dimensions of everyday eating and drinking episodes. Appetite 2007; 48 (2): 218-31.

14. Bellisle F, Blundell J. Satiation, satiety: concepts and organization of behavior. En: Blundell J, Bellisle F, Editores. Satiation, satiety and the control of food intake, theory and practice. Philadelphia: Editorial Woodhead Publishing; 2013. p. 3-11.

15. Bisogni C, Jastran M, Blake C. The construction of eating episodes, food scripts, and food routines. En Preedy V, Watson R, Martin C, Editores. Handbook of behavior, food and nutrition. New York: Editorial Springer; 2011. p. 987-1009.

16. Bourges-Rodríguez H. Los alimentos, la dieta y la alimentación. En Casanueva E, Kaufer-Horwitza M, Perez-Lizaur A, Arroyo P, Editores. Nutriología Médica. D.F., México: Editorial Fundación Mexicana para la Salud, Médica Panamericana; 2008. p. 598-662.

17. Levitsky D. The control of eating: is there any function for satiation and satiety?. En: Blundell J, Bellisle F, Editores. Satiation, satiety and the control of food intake, theory and practice. Philadelphia: Editorial Woodhead Publishing; 2013. p. 373-93.

18. Brondel L, Romer M, Van Wymelbeke V, Pineau N, Jiang T, Hanus C, et al. Variety enhances food intake in humans: Role of sensory-specific satiety. Physiol Behav 2009; 97 (1): 44-51.

19. Chung S, Vickers Z. Influence of sweetness on the sensory-specific satiety and long-term acceptability of tea. Food Qual Pref 2007; 18 (2): 256-64.

20. García-Burgos D, Secchiari F, Calviño A. Is sensory-spe- cific satiety for a bitter sweet infusion modulated by context? Physiol Behav 2015; 140: 180-7.

21. Guinard JX, Brun P. Sensory-specific Satiety: Comparison of Taste and Texture Effects. Appetite 1998; 31 (2): 141-57.

22. Smeets A, Westerterp-Plantenga M. Oral exposure and sensory-specific satiety. Physiol Behav 2006; 89 (2): 281 6.

23. Chapelot D. Quantifying satiation and satiety. En Blundell J, Bellisle F, Editores. Satiation, satiety and the control of food intake, theory and practice. Philadelphia: Editorial Woodhead Publishing; 2013. p. 12-39.

24. Blundell J, De Graaf C, Hulshof T, Jebb S, Livingstone B, Lluch A, et al. Appetite control: methodological aspects of the evaluation of foods. Obes Rev 2010; 11 (3): 25170 .

25. González-Jiménez E, Schmidt Río-Valle J. Regulación de la ingesta alimentaria y del balance energético; factores y mecanismos implicados. Nutr Hosp 2012; 27 (6): 18509.

26. Strang J. Satiety as a factor in nutritional states. Trans Am Clin Climatol Assoc 1950; 62: 41-50.

27. Meyer J. Satiety and Weight Control. Am J Clin Nutr 1957; 5: 184-5.

28. Hollifield G, Parson W. Studies of the satiety responce in mice. J Clin Invest 1957; 36 (12): 1638-41.

29. Booth D. Approaches to Feeding Control. Appetite and food intake 1976; 417-78.

30. Holt S, Brand J, Petocz P, Farmakalidis E. A satiety index of common foods. Eur J Clin Nutr 1995; 49 (9): 675-90.

31. Bellisle F, Drewnowski A, Anderson H, Westerterp-Plantenga M, Martin C. Sweetness, Satiation, and Satiety. J Nutr 2012; 142 (6): 1149S-54S.

32. Dennis EA, Flack KD, Davy BM. Beverage Consumption and Adult Weight Management: A Review. Eat Behav 2009; 10 (4): 237-46.

33. De Wijk R, Zijlstra N, Mars M, de Graaf C, Prinz J.F The effects of food viscosity on bite size, bite effort and food intake. Physiol Behav 2008; 95 (3): 527-32.

34. Mattes R. Fluid calories and energy balance: The good, the bad, and the uncertain. Physiol Behav 2006; 89 (1): 66-70.

35. García-Almeida J, Casado G, García J. Una visión global y actual de los edulcorantes. Aspectos de regulación. Nutr Hosp 2013; 28 (4): 17-31.

36. Low Y, Lacy K, Keast R. The Role of Sweet Taste in Satiation and Satiety. Nutrients 2014; 6 (9): 3431-50.

37. Hill S, Prokosch M, Morin A, Rodeheffer C. The effect of non-caloric sweeteners on cognition, choice, and post-consumption satisfaction. Appetite 2014; 83: 828. 
38. Shankar R, Ahuja S, Sriram K. Non-nutritive sweeteners: Review and update. Nutrition 2013; 29 (11-12): 1293-9.

39. Finlayson G, Bordes I, Griffioen-Roose S, de Graaf C, Blundell J. Susceptibility to Overeating Affects the Impact of Savory or Sweet Drinks on Satiation, Reward, and Food Intake in Nonobese Women. J Nutr 2012; 142 (1): 125-30.

40. Gao J, Xueyan J, Chu-Shern J, Henry J, Zhou W. Influence of bread structure on human oral processing. J Food Eng 2015; 167: 147-55.

41. Richardson P, Saliba A. Personality traits in the context of sensory preference: a focus on sweetness. En Preedy V, Watson R, Martin C, Editores. Handbook of behavior, food and nutrition. New York: Editorial Springer; 2011. p. 85-97.

42. Hogenkamp P, Stafleu A, Mars M, Brunstrom J, De Graaf C. Texture, not flavor, determines expected satiation of dairy products. Appetite 2011; 57 (3): 635-41.

43. De Graaf C. Texture and satiation: the role of oro-sensory exposure time. Physiol Behav 2012; 107 (4): 496501.

44. Muñoz-Cano J, Córdova-Hernández J, Boldo-León X. Ambiente obesogénico y biomarcadores anómalos en escolares de Tabasco, México. Salud en Tabasco 2012; 18 (3): 87-95.

45. Cienfuegos A. Secreción gástrica e inhibidores de bomba de protones. Rev Col Gastroenterol 2010; 25 (1): 94-8.

46. Khoo J, Rayner C, Feinle-Bisset C, Wittert G. Role of the gastrointestinal tract in peptide hormone release and appetite. En Preedy V, Watson R, Martin C, Editores. Handbook of behavior, food and nutrition. New York: Editorial Springer; 2011. p. 1035-49.

47. Szczesniak A. Texture is a sensory property. Food Qual Prefer 2002; 13 (4): 215-25.

48. Keller K, Kral T, Rolls B. Impacts of energy density and portion size on satiation and satiety. En Blundell J, Bellisle F, Editores. Satiation, satiety and the control of food intake, theory and practice. Philadelphia: Editorial Woodhead Publishing; 2013. p. 115-27.

49. Merril E, Kramer F, Cardello A, Schutz H. A comparison of satiety measures. Appetite 2002; 39 (2): 181-3.

50. Mancilla J, Cisneros A, López V, Ocampo M, Alvarez G, Vázquez R, et al. Efecto del 5-HdlTP: un análisis microestructural de la conducta alimenticia. Rev Mex Anal Conducta 1993; 19 (1-2): 3-18.

51. Harrold J, Halford J. Manipulation of diet to alter appetite. En Preedy V, Watson R, Martin C, Editores. Handbook of behavior, food and nutrition. New York: Editorial Springer; 2011. p. 1051-67.

52. Wansink B, Van Ittersum K, Painter J. How Diet and Health Labels Influence Taste and Satiation. J Food Sci 2004; 69 (9): S340-6.

53. Llewellyn C, Wardle J. Satiation and satiety in obesity. En Blundell J, Bellisle F, Editores. Satiation, satiety and the control of food intake, theory and practice. Philadelphia: Editorial Woodhead Publishing; 2013. p. 298-315. 